\title{
Comment on Williams NH, et al.: a systematic review and meta- analysis of biological treatments targeting tumour necrosis factor $\alpha$ for sciatica (Eur Spine J, 2013;22(9):1921-35)
}

\author{
Jing Wang $\cdot$ Gui-tao Li $\cdot$ Hong-tao Sun
}

Received: 10 December 2013/Revised: 2 January 2014 / Accepted: 4 January 2014/ Published online: 17 January 2014

(c) Springer-Verlag Berlin Heidelberg 2014

To the Editor,

In this system review, Williams et al. [1] compared biological agents, targeting tumour necrosis factor $\alpha$ (TNF- $\alpha$ ), for sciatica with placebo and alternative interventions. They found that there was a reduction in the need for discectomy, which was not statistically significant, and no difference in the number of adverse effects. It is a very valuable study. Nevertheless, there are some questions we would like to ask related to this article.

To begin with, the investigators (Williams et al.) included five randomised controlled trials (RCTs) and one non-RCT of adults who had sciatica treated by biological agents compared with placebo or alternative interventions. In our opinion, the non-RCT [2,3] should be excluded from the system review to enhance the credibility.

Then, the investigators used a random-effect model in all comparisons, even though there was no heterogeneity in leg pain intensity (Fig. 5), needing disc surgery (Fig. 7) and adverse effects (Fig. 8). We suggest that a fixed-effect model should be used to pool the results.

Furthermore, it is not appropriate that summary odds ratio (OR) and standard mean difference (MD) estimates with corresponding $95 \%$ CIs were derived by using the method of Mantel-Haenszel $(\mathrm{M}-\mathrm{H})$ or Inverse variance (IV) with the assumptions of a random-effects model. We

\section{J. Wang}

Department of Orthopedic Surgery, The Affiliated Southern Guangdong Hospital of Southern Medical University,

Guangzhou 510317, People's Republic of China

\section{J. Wang · G. Li $(\bowtie) \cdot$ H. Sun}

Department of Orthopedic Surgery, Guangdong No. 2 Provincial

People's Hospital, Guangzhou 510317,

People's Republic of China

e-mail: guitao2013@126.com suggest that "DerSimonian and Laird random effect model" should be used to evaluate globe effect (Fig. 2), leg pain intensity (Fig. 3) and Oswestry Disability Index (Figs. 4, 6).

Eventually, the investigators did not assess the publication bias. In our opinion, the publication bias should be assessed by funnel plots and statistical tests (e.g., Egger's linear regression test or Begg's rank correlation test).

We agree that biological agents are expensive but may lead to cost savings and alongside RCTs are needed to assess economic evaluations. In our opinion, further large RCTs are needed to establish the efficacy of TNF- $\alpha$ compared with placebo.

Acknowledgments This work was supported by funding of Science \& Technology Planning Project of Guangdong Province, People's Republic of China, NO.2012B031800266.

Conflict of interest We have no competing interests to declare.

\section{References}

1. Williams NH, Lewis R, Din NU, Matar HE, Fitzsimmons D, Phillips CJ, Sutton A, Burton K, Hendry M, Nafees S, Wilkinson C (2013) A systematic review and meta-analysis of biological treatments targeting tumour necrosis factor $\alpha$ for sciatica. Eur Spine J 22(9):1921-1935

2. Karppinen J, Korhonen T, Malmivaara A, Paimela L, Kyllönen E, Lindgren K-A, Rantanen P, Tervonen O, Niinimäki J, Seitsalo S, Hurri H (2003) Tumor necrosis factor-alpha monoclonal antibody, infliximab, used to manage severe sciatica. Spine 28:750-754

3. Korhonen T, Karppinen J, Malmivaara A, Autio R, Niinimäki J, Paimela L, Kyllönen E, Lindgren K-A, Tervonen O, Seitsalo S, Hurri H (2004) Efficacy of infliximab for disc herniation induced sciatica; one year follow-up. Spine 29:2115-2119 